\title{
MicroRNA-19b targets Mfn1 to inhibit Mfn1-induced apoptosis in osteosarcoma cells
}

\author{
X. LI' ${ }^{1}$, F. S. WANG ${ }^{2}$, Z. Y. WU' ${ }^{2}$ J. L. LIN ${ }^{2}$, W. B. LAN ${ }^{1}$, J. H. LIN ${ }^{2, *}$ \\ ${ }^{1}$ The First Clinical Medical College of Fujian Medical University, Fuzhou 350004, China; ${ }^{2}$ Department of Orthopedics, The First Affiliated Hospital \\ of Fujian Medical University, Fuzhou 350004, China \\ ${ }^{*}$ Correspondence: Jianhuafmu@163.com
}

Received August 1, 2013 / Accepted September 27, 2013

\begin{abstract}
Accumulative evidence has confirmed that, miR-17-92, a typical polycistronic mRNA cluster, was up-regulated in various solid tumors, and play an important role in the occurrence and development progress of tumors. In our study, we detected the six members of miR-17-92 cluster in osteosarcoma cell line, finding that the expression of miR-17 and miR-19b was up-regulated significantly. Further studies have found that Mfn1 was one of the target genes of miR-19b and the transcription and expression level of Mfn1 were down-regulated by miR-19b. MTS, flow cytometry, TUNEL-DAPI, Annexin V-FITC and transwell assay demonstrated that Mfn1 significantly blocked the cell cycle, promoted apoptosis and inhibited proliferation and invasion of osteosarcoma cells. Whereas, miR-19b targets 3'UTR sequences of Mfn 1 genes inhibit the expression of Mfn1, thus inhibited Mfn1 triggered anti-cancer effect. Taken together, miR-19b functions by targeting Mfn1 reduce the protein expression level, thus provides a novel target to understand the molecular biology and genetics mechanisms of occurrence and development of osteosarcoma, contributing to the diagnosis and therapy of osteosarcoma.
\end{abstract}

Key words: Mfn1, miR-19b, osteosarcoma, cell cycle arrest, apoptosis

Osteosarcoma, one of the most common malignant bone tumors, possesses $34 \%$ of the primary malignant bone tumors, which is one of the five malignant tumors in teenagers and young adults [1]. For high degree of malignancy and poor prognosis of osteosarcoma, five-year survival rate of patients treated with operation combined with chemotherapy treatments was only 55-68\% [2]. The occurrence of osteosarcoma, as a complex process with polygenes and multi-steps, was of limited cognition in the area of molecular mechanism.

Recent years, miRNA, a vital gene regulatory molecules in organism, has kindled great interests in the relationships with disease especially cancers in the biomedical domain. Under the unremitting efforts of biological workers, progresses have been made on miRNA functions. Thereinto, miR-17-92 cluster is of in-depth exploration. MiR-17-92, a typical polycistronic miRNA cluster, locates on the third intron of a $7 \mathrm{~kb}$ primary transcript C13orf25 in the chromosome 13q31.3 and encodes six highly conserved mature miRNAs (miR-17, miR-18a, miR-19a, miR-20a, miR-19b-1 and miR-92-1), which plays an important role in the normal development of lung, heart and immune system as well as tumorigenesis [3]. Accumulative researches showed that miR-17-92 cluster and relative collateral homologous sequences were apparently up-regulated in a variety of solid tumors, which were of great significance in the occurrence and progress of tumors [4-6]. Baumhoer et al. [5] utilized miRNAarray to examine miRNA expression profiles in six osteosarcoma cell lines (HOS58, U2-OS, Saos2, MNNG/HOS, SJSA-1 and MG-63), cultured osteoblasts (hFOB 1.19) and mesenchymal stem cells (L87/4) miRNAs were down-regulated in the majority of osteosarcoma cell lines and several miRNAs with oncogenic and tumor suppressor properties, including various members of the oncogenic miR-17-92 cluster were identiefied.

Mitofusins (Mfn1 and Mfn2) are human homologues of the only known protein mediators of mitochondrial fusion, playing important mechanistic roles in the regulation of mitochondrial fusion and maintenance of dynamic balance of mitochondrial morphology [7]. Mfn1 majorly promotes the combination of mitochondrion in the early period of the fusion and Mfn2 mainly functions in the later period of 
mitochondrial fusion, promoting mitochondria fusion and docking of inner membrane $[8,9]$. Meanwhile, numbers of studies revealed that the fusion and fission of mitochondria was closely related with cell apoptosis. Overexpression of Mfn1 and Mfn2 facilitates mitochondrial fusion and cell apoptosis, while inhibition of Mfn1 and Mfn2 expedites the growth of tumor cells [10]. According to the low expression of Mfn2 in bladder cancer tissues, Jin et al. [11] speculated and confirmed the distinct anti-proliferation and apoptosis-promoting effect of Mfn2 in the bladder cancer cell line, finding that increase of Mfn2 expression level led to cell growth arrest, generation of activated caspase- 3 and lysis of poly A (DP-ribose) polymerase (PARP), thus promoted cell apoptosis. It was reported that overexpression of Mfn2 in recombinant adenovirus vector redounded to the apoptosis of a wide variety of tumor cell lines such as hepatoma carcinoma cells, Hela, HT-29 and MCF-7, which was verified by in vivo and in vitro nude mouse tumorigenicity assay [12]. However, investigation on Mfn1 is still currently.limited.

In the present study, we examined the expression levels of the six members in miR-17-92 cluster in MG-63, Saos-2, U2-OS and hFOB 1.19 cells, finding that miR-17, miR-19b, miR-20a and miR-19a were significantly up-regulated in osteosarcoma cells, of which miR-17 and miR-20a were of similar functions $[4,13]$ and miR-19b and miR-19a acted similarly [14]. Since the effect of miR-20a in osteosarcoma cells has been reported previously [4] while studies related to miR-19b functions in osteosarcoma cells are limited, miR-19b is selected for the further investigations. MiRBase, miRanda and TargetScanHuman 6.2 were exploited to analyze the target genes of miR-19b, finding that 3' UTR regions on the Mfn1 and Mfn2 genes acted as favorably matched sites, of which Mfn1 was of the highest degree of match. Furthermore, we elucidated the relationship between miR-19b and Mfn 1 to validate that Mfn1 acts as the target gene of miR-19b, and explored the regulation molecular of mechanisms in osteosarcoma cells.

\section{Materials and methods}

Cell culture. Osteosarcoma cell line (MG-63, U2-OS, and Saos-2) and cultured osteoblasts (hFOB 1.19) were derived from American Type Culture Collection (ATCC) and cultured with $10 \%$ fetal bovine serum and $2 \%$ L-glutamine (Invitrogen) containing RPMI 1640 (Invitrogen, Karlsruhe, Germany). The cells were maintained at $37^{\circ} \mathrm{C}$ in an atmosphere of humidified air with $5 \% \mathrm{CO}_{2}$ in a cell culture incubator.

Vector construction and transfection. RNA was extracted from hFOB 1.19 cells with Trizol (Invitrogen) according to the instructions of the manufacturer, followed by reverse transcription PCR to amplify the coding region of Mfn 1 . Then the products were digested with BamH I and EocRI (TaKaRa), cloned into pcDNA3.1 vectors, sequenced and verified. The primers used for Mfn 1 amplification were Mfn 1 Bam H IF (5'CGGGATCCGCCACC ATGGCAGAACCTGTTTCTCC-3') and Mfn 1 EocRIR (5'-CGGAATTCTTAGGATTCTTCATTGCTTCAAGG-3').

The cells were seeded into 6 -well plate at $1 \times 10^{5} \mathrm{cells} / \mathrm{mL}$ and incubated for $24 \mathrm{~h}$. Plasmid, hsa-miR-19b mimic, mimic negative control, hsa-miR-19b inhibitor and inhibitor negative control (RiboBio) transfection were carried out following the instruction of Lipofectamine2000 (Invitrogen) when the cell confluence reached about $70 \%$. The concentration of plasmid was $2 \mu \mathrm{g} /$ well and hsa-miR-19b mimic, mimic negative control, hsa-miR-19b inhibitor and inhibitor negative control were $50 \mathrm{nM} /$ well. After $72 \mathrm{~h}$, transfected cells were analyzed by real-time PCR and Western blotting.

Fluorescence quantitative PCR. Total RNA was extracted from the cells using Trizol. Stem-loop qRT-PCR assay was performed with $1 \mu \mathrm{L}$ RNA and $1 \mu \mathrm{l}$ specific stem-loop RT primers $(10 \mu \mathrm{M})$ for $5 \mathrm{~min}$ at $85^{\circ} \mathrm{C}$ followed by ice cool. $4 \mu \mathrm{l} 5 \times$ buffer, $2 \mu \mathrm{ldNTPs}(10 \mathrm{mM}), 0.5 \mu \mathrm{l}$ RNase inhibitor and $0.5 \mu \mathrm{l} \mathrm{M}-\mathrm{MLV}$ (Promega) were added with total volume of $20 \mu \mathrm{l}$. The reaction was performed at one cycle at $42^{\circ} \mathrm{C}$ for $60 \mathrm{~min}$, one cycle at $85^{\circ} \mathrm{C}$ for $10 \mathrm{~min}$ and preserved at $4^{\circ} \mathrm{C}$. Quantitative PCR was

Table 1 Primers for quantitative PCR

\begin{tabular}{llcc}
\hline Name & Primer sequences & Product sizes (bp) & Accession No. \\
\hline hsa-miR-17 & F: 5'-ACACTCCAGCTGGGCAAAGTGCTTACAGTGCA-3' & 73 & MIMAT0000070 \\
hsa-miR-18a & F: 5'-ACACTCCAGCTGGGTAAGGTGCATCTAGTGCAGA-3' & 73 & MIMAT0000072 \\
hsa-miR-19a & F5'-ACACTCCAGCTGGGTGTGCAAATCTATGCAAA-3' & 93 & MIMAT0000073 \\
hsa-miR-19b & F: 5'-ACACTCCAGCTGGGTGTGCAAATCCATGCAAA-3' & 93 & MIMAT0000074 \\
hsa-miR-20a & F: 5'-ACACTCCAGCTGGGTAAAGTGCTTATAGTGCA-3' & 73 & MIMAT0000075 \\
hsa-miR-92a & F: 5'-ACACTCCAGCTGGGTATTGCACTTGTCCCGGCCTG-3' & 72 & MIMAT0000092 \\
miRNA-R & 5'-CTCAACTGGTGTCGTGGA-3' & & NR_002752 \\
U6 & F: 5'-CTCGCTTCGGCAGCACA-3' & 94 & NM_002046 \\
GAPDH & R: 5'-AACGCTTCACGAATTTGCGT-3' & & 128 \\
MFN1 & F: 5'-GGTATCGTGGAAGGACTC-3' & & NM_033540 \\
\hline
\end{tabular}


$20 \mu \mathrm{l}$ of total volume, including $5 \mu \mathrm{l}$ cDNA (1:20 dilution), 10 $\mu \mathrm{l} 2 \times$ SYBR Green PCR Master Mix (Toyobo), $0.5 \mu$ forward primer, $0.5 \mu \mathrm{l}$ reverse primer, and $4 \mu \mathrm{l}$ RNase-free ddH2O. Primers are listed in Supplementary Table 1. The reaction was performed using ABI 7300 quantitative RT-PCR at $50^{\circ} \mathrm{C}$ for $2 \mathrm{~min}$ and $95^{\circ} \mathrm{C}$ for $2 \mathrm{~min}$, followed by 40 cycles of $95^{\circ} \mathrm{C}$ for $15 \mathrm{~s}$ and $60^{\circ} \mathrm{C}$ for $32 \mathrm{~s}$. U6 snRNA was utilized as internal control for the detection of miRNAs and primers were purchased from Ambion. Meanwhile, oligodT primer was used for reverse transcription and SYBR Green PCR Master Mix (Toyobo) was prepared for the detection of Mfn1. Reactions were performed at 40 cycles of $95^{\circ} \mathrm{C}$ for $5 \mathrm{~min}, 95^{\circ} \mathrm{C}$ for $30 \mathrm{~s}$, $55^{\circ} \mathrm{C}$ for $30 \mathrm{~s}$ and $72^{\circ} \mathrm{C} 30$ s. Three independent experiments were conducted for each sample. Data were analyzed by comparing the $2-\Delta \Delta$ Ct value.

Western blot analysis. Total cellular proteins were extracted by incubating cells in lysis buffer. The protein concentrations in the cell lysates were determined by BCA Protein Assay Reagent Kit (Pierce). SDS-PAGE was done in $8 \%$ glycine gels (Bio-rad) loading equal amount of proteins per lane. After electrophoresis, separated proteins were transferred to nitrocellulose membrane and blocked with 5\% non-fatmilk in TBST buffer for $1 \mathrm{~h}$. After that, the membranes were incubated with Mfn1 (Novus Biologicals) at 1:500 dilutions, Mfn2 (Cell Signaling Technology) at 1:1000 dilutions and GAPDH antibodies (Novus Biologicals) at 1:1000 dilutions in $5 \%$ non-fat milk overnight at $4^{\circ} \mathrm{C}$, and then anti-rabbit IgG monoclonal antibody conjugated at room temperature with horseradish peroxidase (southern biotech) at 1:2000 dilution for $1 \mathrm{~h}$. Protein bands were detected using the West Femto system (Pierce).

Dual luciferase assay. The full-length Mfn 1 3'UTR was amplified from cDNA using forward primer F1 (Table 2) and the reverse primer R1. Two paired primers (F1 plus mutR1 and mutF1 plus R1) were used to generate mutant-type Mfn1 3'UTR in which the four mutant nucleotides were underlined within the seed region of miR-19b binding site by PCR-based mutagenesis method. Both PCR fragments were cloned into psiCHECK-2 vector (Promega). Reporter vector psiCHECK-2 carrying the 3'UTR sequences of Mfn 1 were assayed for luciferase expression using the Dual Glo Luciferase Assay System (Promega) following the manufacturer's instructions. The relative expression of target-specific Renilla luciferase was determined by taking the normalized levels compared with background firefly luciferase for each sample transfected a minimum of three replicates \pm stand- ard error and is representative of at least two independent experiments.

MTS cell proliferation assay. Cells were seed into 96-well plate at $1 \times 10^{5}$ cells with $100 \mu \mathrm{l}$ per well and collected at different time points (0h, 24h, 48h, 72h). 10 $1 \mathrm{l}$ MTS (Promega) per $100 \mu \mathrm{l}$ were added for $4 \mathrm{~h}$ incubation, followed by microplate reader determination with OD 490.

TUNEL-DAPI co-staining assay. Apoptotic cells were detected using TUNEL assay following the manufacturer's protocol. Cells were fixed with $4 \%$ formaldehyde for $25 \mathrm{~min}$ at $4^{\circ} \mathrm{C}$ and lysed with $0.2 \%$ Triton X-100 in PBS for $5 \mathrm{~min}$ at room temperature. The cells were cultured with TUNEL reaction mixture for $1 \mathrm{~h}$. For nuclear staining, cells were incubated with $0.5 \mu \mathrm{g} / \mathrm{mL}$ of DAPI for $5 \mathrm{~min}$ at $37^{\circ} \mathrm{C}$. At the end of incubation, the cells were rinsed with PBS and observed under a fluorescence microscope.

Annexin V-FITC apoptosis analysis. Cells were collected after transfection for $72 \mathrm{~h}$, and the translocation of phosphatidylserine in treated cells was detected using the Annexin-V-FITC staining kit (keygen). Briefly, cells were suspended in $500 \mu \mathrm{L}$ of binding buffer and incubated at room temperature in the dark for $15 \mathrm{~min}$ after labeled with $1.25 \mu \mathrm{L}$ of Annexin V-FITC and $10 \mu \mathrm{L}$ of propidium iodide. The stained cells were then analyzed by flow cytometry.

Flow cytometric analysis. $2 \times 10^{5}$ cells were seed into 6 -well plate and transfected after $24 \mathrm{~h}$ culture. $48 \mathrm{~h}$ later, cells were harvested and fixed with pre-cooled $70 \%$ ethanol overnight at $4^{\circ} \mathrm{C}$ overnight. The fixed cells were washed with PBS and stained with propidium iodide (PI) for $30 \mathrm{~min}$ at $4^{\circ} \mathrm{C}$. The stained cells were determined by flow cytometer to explore cell cycle distribution, followed by data analysis using ModFit software.

Transwell matrigel invasion assay. Invasion of cells was evaluated by Transwell matrigel invation assay. Briefly, 200 $\mu \mathrm{L}$ of cells after transfection $\left(1 \times 10^{6}\right.$ cells $\left./ \mathrm{ml}\right)$ and $600 \mu \mathrm{L}$ of the complete medium were added to the upper and lower compartments of the chamber respectively. After incubation of $48 \mathrm{~h}$, cells migrating to the lower side of the filter were fixed with $4 \%$ paraformaldehyde for $15 \mathrm{~min}$ at room temperature, washed with PBS, stained with crystal violet, and then observed under a confocal microscope.

Statistical analysis. Experiments were carried out at least in triplicate and results were expressed as mean \pm S.D. Statistical analysis was performed using SPSS statistical program version 17.0. Difference with $P<0.05\left({ }^{*}\right)$ or $P<0.01\left({ }^{* *}\right)$ was considered statistically significant. Cell reproductive capac-

Table 2 Primers for Mfn1 3'UTR amplification

\begin{tabular}{ll}
\hline Name & Primer sequences \\
\hline F1 & 5'-CCGCTCGAG CAATAGAGATTGCTTTGGTGAC-3’ \\
R1 & 5'-ATAAGAATGCGGCCGC CATCAGTTAAAGATTTTATTTGATTCA-3’ \\
mutR1 & 5'-ATGAATGACGTCTCGGAGGCAAAACTGGCCTC-3' \\
mutF1 & 5'-TGCCTCCGAGACGTCATTCATTTCAGTTATTTCTGATCC-3’' \\
\hline
\end{tabular}




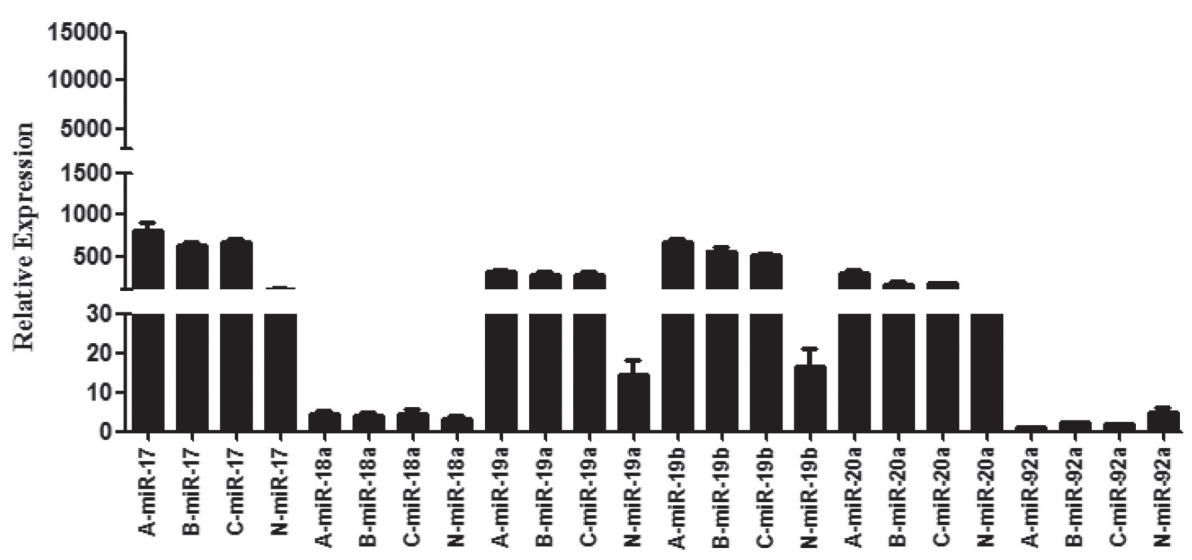

Figure 1. Expression levels of miR-17-92 gene family members in osteosarcoma cells as determined by fluorescence quantitative RT-PCR. A: MG-63 cells; B: Saos-2 cells; C: U2-OS cells; N: hFOB 1.19 cells.

ity, apoptosis assay and invasion assay were analyzed by FD ANOVA multiple comparisons. Fluorogenic quantitative PCR and Western Blotting were analyzed by one-way ANOVA multiple comparisons.

\section{Results}

Expression levels of miR-17-92 cluster family members in osteosarcoma cells. Fluorescence quantitative RT-PCR was utilized to examine the expression levels of the six members of miR-17-92 cluster in MG-63, Saos-2, U2-OS and hFOB 1.19 cells, finding that miR-17, miR-19b, miR-20a and miR-19a were significantly up-regulated in osteosarcoma cells (Fig. 1), of which miR-17 and miR-20a were of similar functions and miR-19b and miR-19a acted similarly. Since the effect of miR-20a in osteosarcoma cells has been reported previously while studies related to miR-19b functions in osteosarcoma cells are limited, miR-19b is selected for the further investigations.

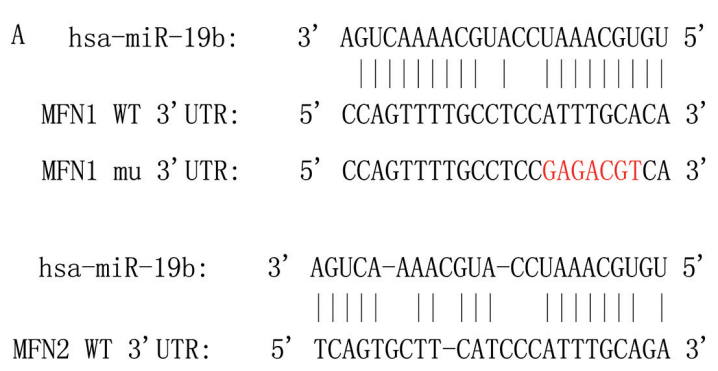

Mfn 1 acts as one of the target genes of miR-19b. MiRBase, miRanda and TargetScanHuman 6.2 were exploited to analyze the target genes of miR-19b, finding that 3' UTR regions on the Mfn1 and Mfn2 genes acted as favorably matched sites, of which Mfn1 was of the highest degree of match (Fig. 2a). Hence, dual-luciferase reporter assay system was used to verify the existence of miR-19b target site on Mfn1 genes. Firstly, 3'UTR sequences of Mfn 1 genes were cloned into the dual luciferase reporter vector psiCHECK-2 and target sites of miR$19 \mathrm{~b}$ were mutated. Then cloned Mfn 1 3'UTR vector, mutant Mfn1 3'UTR vector and miR-19b mimics were respectively transfected into HEK-293T, and the change in expression of luciferase was analyzed. Taking cells transfected with reporter vector as a control, luciferase expression level of the cells transfected with cloned Mfn1 3'UTR vector significantly decreased about $36 \%$, while luciferase expression of the cells transfected with mutant Mfn1 3'UTR vector showed no apparent change (Fig. 2b). Taken together, these results validate that miR-19b could target Mfn1 3'UTR region.

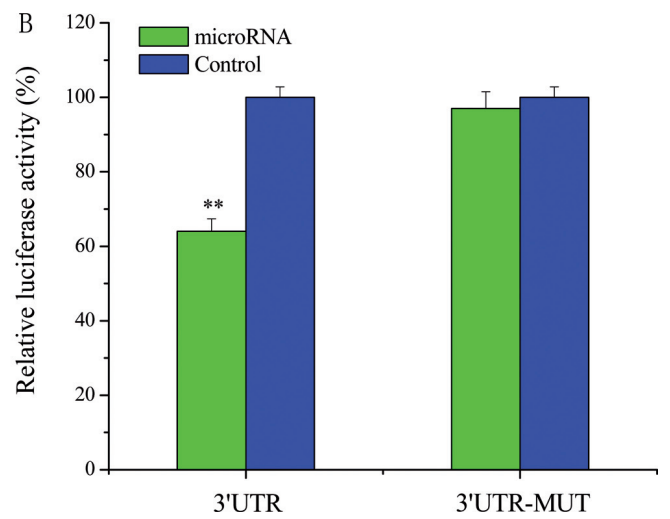

Figure 2. Prediction of miR-19b target gene. (a) miR-19b target sites in the conservative sequence of Mfn1 and Mfn2 3'UTR. (b) The luciferase expression level of HEK-293T cells transfected with cloning Mfn1 3'UTR vector, mutant Mfn1 3'UTR vector and miR-19b mimics. Each bar represents the mean \pm SD from three samples $\left({ }^{*} P<0.01\right)$. 

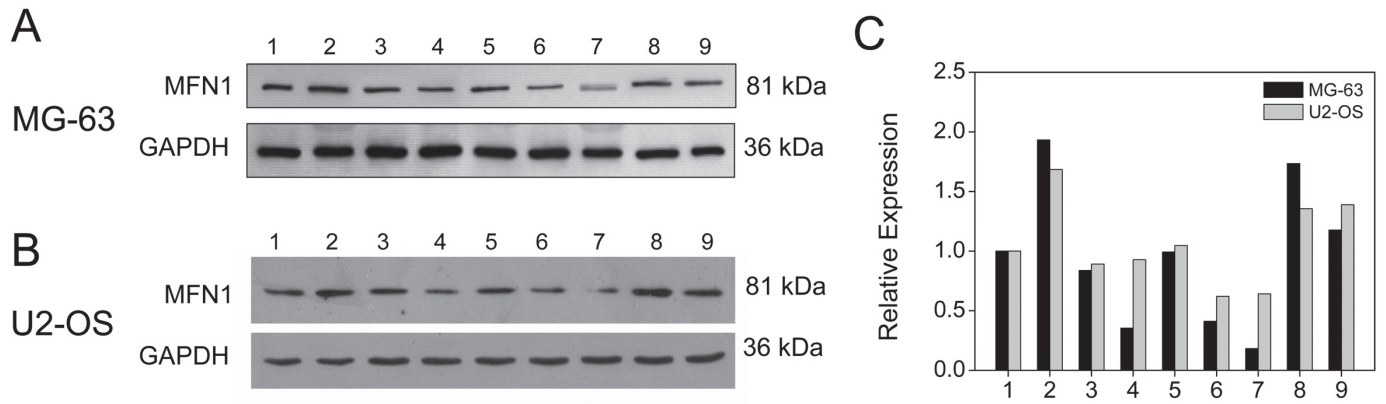

Figure 3. Expression levels of MFN1 as examined by Western blotting. 1. Control cells, 2. hsa-miR-19b inhibitor, 3. inhibitor Negative Control (iNC), 4. hsa-miR-19b mimic+pcDNA3.1 vector, 5. mimic Negative Contro $1(\mathrm{mNC})$, 6. hsa-miR-19b mimic+pcDNA3.1-MFN1, 7. hsa-miR-19b mimic, 8 . pcDNA3.1-MFN1 and 9. pcDNA3.1+.

MiR-19b inhibits Mfn1 expression in osteosarcoma cells. For the examination of interaction between Mfn 1 and miR$19 \mathrm{~b}$ in osteosarcoma cells, mimics and inhibitors of miR-19b were synthesized and overexpression vectors of Mfn1 were constructed to transfect into MG-63 and U2-OS cells. As shown by Western blot analysis (Fig. 3), Mfn1 expression was sharply down-regulated as the transfection of miR-19b mimics but increased as the addition of miR-19b inhibitors, proving that miR-19b inflicted inhibitory effect on the expression of Mfn1.

MiR-19b inhibits Mfn1-induced growth inhibition in osteosarcoma cells. Considering that miR-19b targeted 3'UTR region of Mfn 1 gene to down-regulate the expression of Mfn 1 , we examined the influence of such down-regulated effect on osteosarcoma cells. MTS cell proliferation assay was conducted to explore the impact on MG-63 cell growth (Fig. 4). It was showed that cell proliferation was significantly increased with the transfection of miR-19b mimics but was decreased with the addition of miR-19b inhibitors, revealing that miR-19b might promote the growth of tumor cells. Besides, cell proliferation of the group transfected with both miR-19b mimics and Mfn 1 overexpression vectors (pcDNA3.1-MFN1) differed slightly with the control group, while cell growth of the group

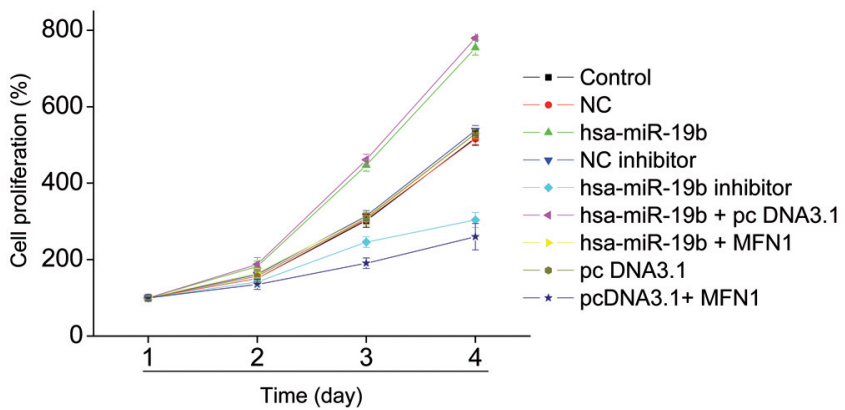

Figure 4. Effects of Mfn1 and miR-19b on osteosarcoma cell proliferation.

transfected with Mfn1 vectors alone was inhibited apparently, suggesting the proliferation-inhibiting effect of Mfn1. Taken together, we may infer that miR-19b targets Mfn1 to suppress the anti-proliferative effect exerted by Mfn1.

Inhibition of miR-19b enhances Mfn1-induced cell cycle arrest and apoptosis. Resistance to apoptosis is a common characteristic of cancer cells. Therefore, the role of Mfn 1 and miR-19b in osteosarcoma development and malignance was examined.
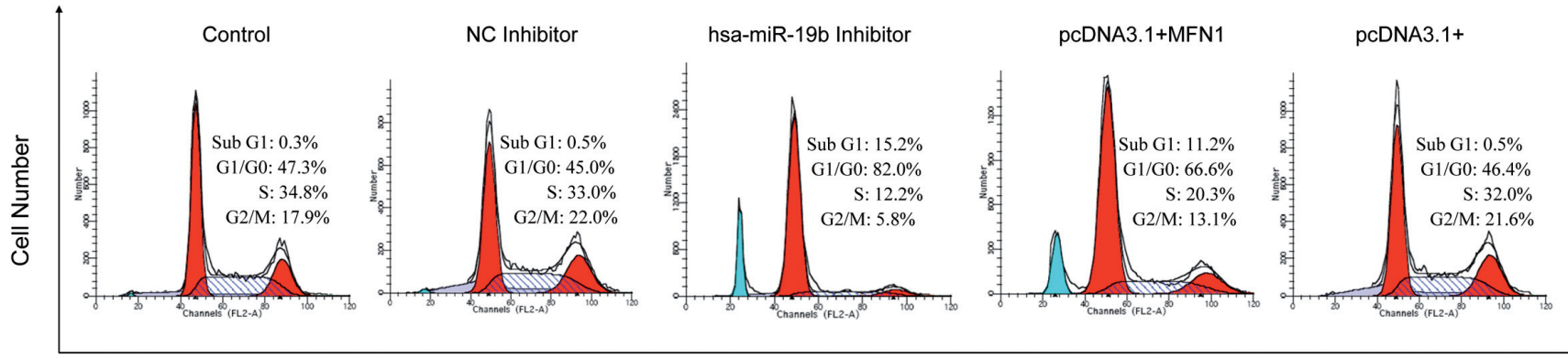

DNA Content

Figure 5. Effects of miR-19b and Mfn1 on the MG-63 cell cycle progression. After treatment with miR-19b inhibitor and Mfn1 vector respectively, the cells were labeled with PI staining solution and the percentage of cell populations in G0/G1, S and G2/M phases was analyzed by flow cytometry. The data represents the mean \pm SD of three independent experiments. 

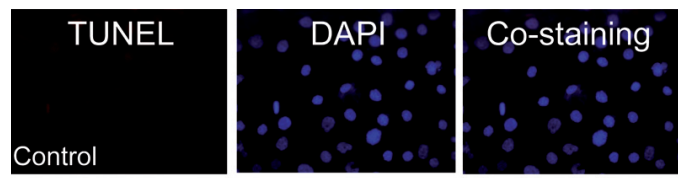

Apoptosis
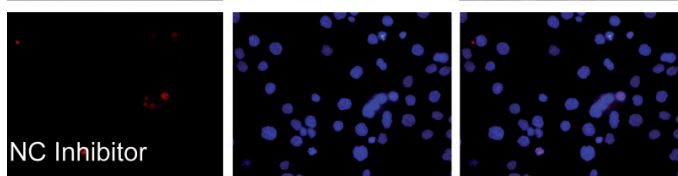

$1 \%$
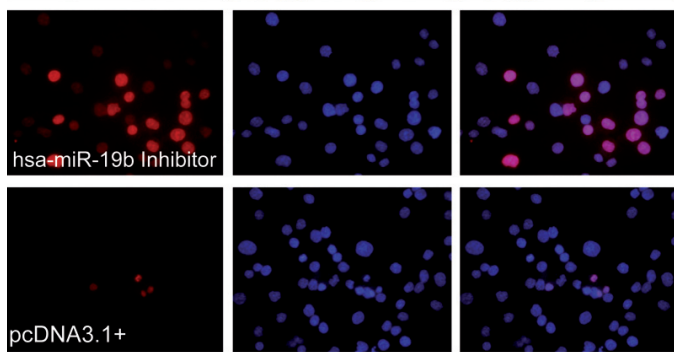

$26 \%$
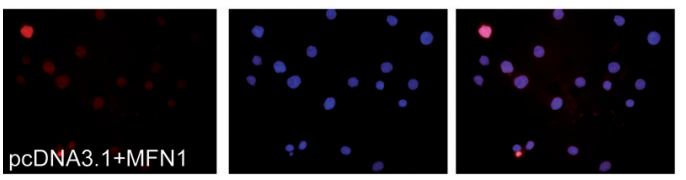

$3 \%$

Figure 6. Examination of osteosarcoma cell apoptosis by TUNEL-DAPI co-staining assay. The images shown here are representative of three independent experiments with similar results.
Flow cytometric assay indicated that the DNA content of G1/G0 phase was increased markedly from $47.3 \%$ to $82.0 \%$ with the addition of miR-19b inhibitor and to $66.6 \%$ with the transfection of Mfn1 vectors. Meanwhile, sub G1 peaks of miR-19b inhibitor group raised from $0.3 \%$ to $15.2 \%$ and sub G1 peaks of Mfn 1 vector group raised from $0.3 \%$ to $11.2 \%$, suggesting a cell arrest at G1/G0 phase accompanied with slight cell apoptosis (Fig. 5). TUNEL-DAPI co-staining assay showed DNA fragmentation and morphological changes of nuclei in the miR-19b inhibitor group and Mfn 1 vector group, implying that the reduction of miR-19b and increase of Mfn 1 both promoted the apoptosis of tumor cells (Fig. 6). Moreover, Annexin VFITC apoptosis assay conducted in U2-OS cells demonstrated that transfection of Mfn 1 vectors and miR-19b inhibitor both significantly triggered early and late apoptosis, and total apoptotic cell death (Fig. 7). Thereinto, late apoptosis induced by Mfn 1 vectors and early apoptosis triggered by miR-19b inhibitor played the leading roles. Taken together, inhibition of miR-19b led to the up-regulation of Mfn1 expression, thus enhanced Mfn1-induced cell arrest and apoptosis.

Mfn1 inhibits the invasion and metastasis of osteosarcoma cells. Malignant cancer cells can acquire the ability of invasion and metastasis. The transwell assay was used to determine the effect of Mfn1 and miR-19b on MG-63 cell invasion (Fig. 8). The results showed that cell invasion was
A

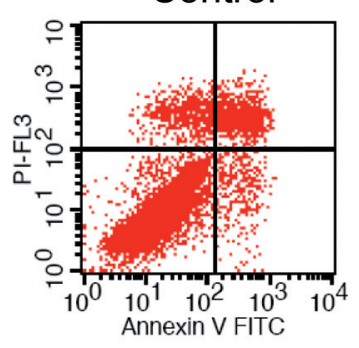

NC Inhibitor

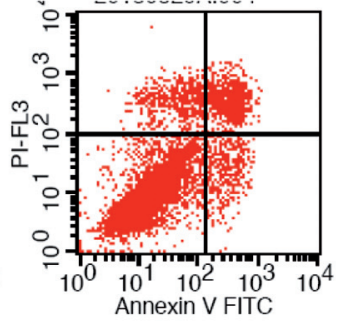

pcDNA3.1+

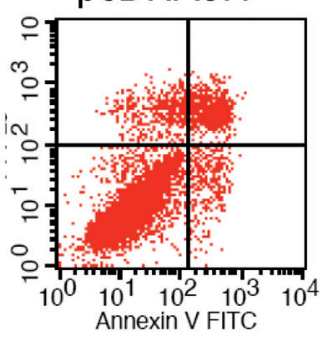

pcDNA3.1+MFN1

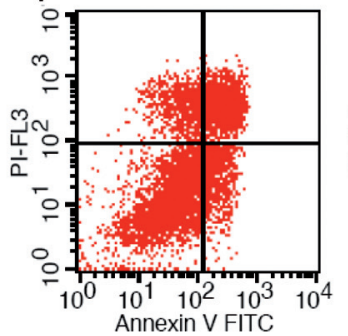

miR-19b Inhibitor

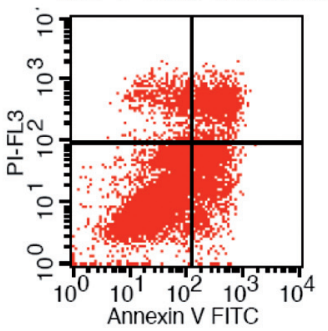

B

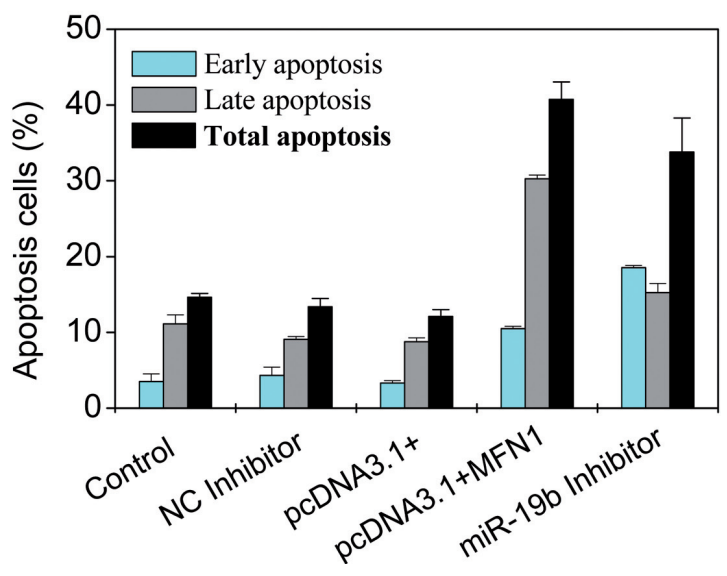

Figure 7. Effects of miR-19b and Mfn1 on osteosarcoma cell apoptosis as measured by Annexin V-FITC-propidium iodide flow cytometric analysis. The results are presented as mean $(n=3) \pm S D,{ }^{* *} P<0.01$ vs. the control. 
A

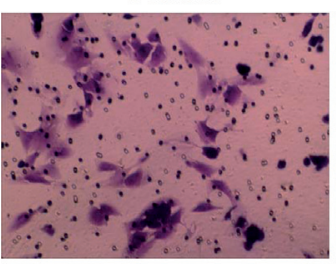

pcDNA3.1+MFN1

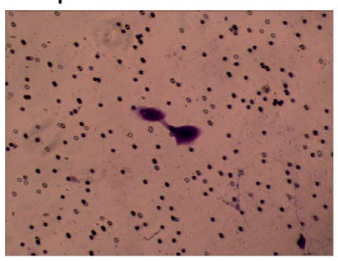

NC Inhibitor

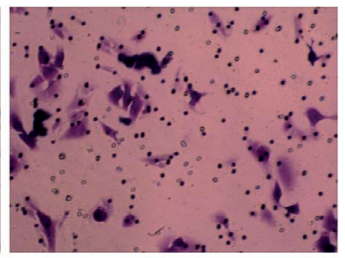

miR-19b Inhibitor

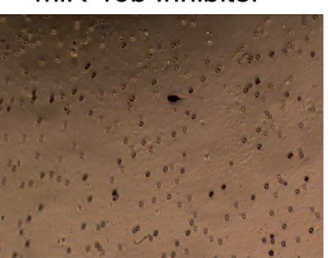

pcDNA3.1+
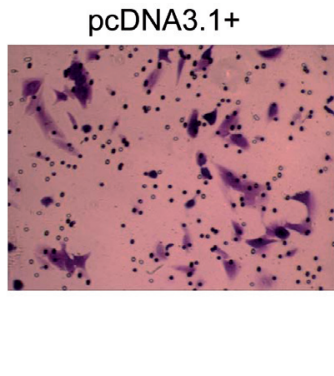

B

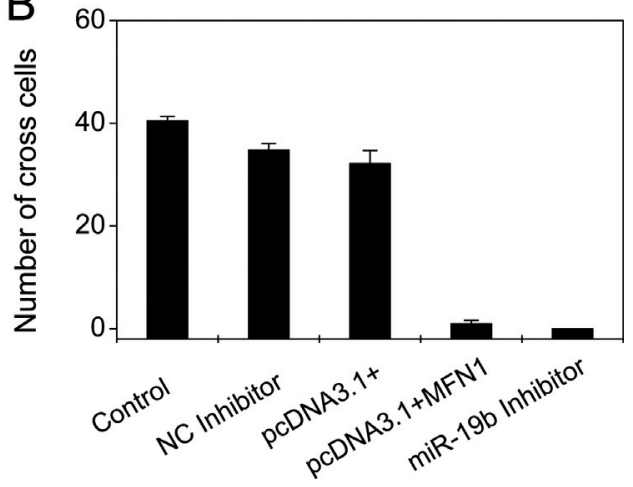

Figure 8. Effects of miR-19b and Mfn1 on osteosarcoma cell invasion as measured by Transwell assay. The results are presented as mean $(n=3) \pm \mathrm{SD}$, ${ }^{*} \boldsymbol{P}<0.01$ vs. the control.

dramatically inhibited by Mfn1, indicating that the Mfn1 played important roles in the invasion of MG-63 cells. Similarly, with the treatment of miR-19b inhibitor, cell invasion and metastasis were almost completely suppressed. Synthetically, we may deduce that the raise of Mfn1 amount caused by inhibition of miR-19b or Mfn1 vector transfection enhances the anti-invasion effect.

\section{Discussion}

Osteosarcoma, one of the most common malignant bone tumors, shows high malignancy and poor prognosis even treated with operation combined with chemotherapy treatment [15]. Genetic level treatment has always been a special interest in the study of osteosarcoma and seeking of effective therapeutic target is the key point of researches [16]. Current studies suggest that miRNA exerts extensive gene regulation functions from all levels of gene activities, being involved in a series of biological processes such as embryonic development, cell proliferation, cell apoptosis and energy metabolism [17-19]. Researches have found that biological behaviors of osteosarcoma closely related to certain miRNA family members in the occurrence and development process [20,21].

MicroRNA (miRNA), a kind of 19 26 nt endogenous non-coding small molecule RNA, regulates gene expression by sequence-specific translation inhibition or mRNA cleavage and participates in a series of important biological processes such as cell development, proliferation, differentiation and apoptosis [22]. As an important gene regulatory molecule, miRNA has kindled great research interests in the biomedical domain on its relationship with diseases especially tumors. Studies have found that various miRNAs play significant roles in the occurrence, development and metastasis processes of malignant cancer through adjustment of target genes and protein expression under different conditions [23, 24]. Sequence analysis of tumor related gene regions in the genome reveals that $52.5 \%$ miRNA encoding genes located around, suggesting the regulation effects of miRNAs on cancer-related genes [25]. MiR-17-92, a typical polycistronic miRNA cluster, locates on the third intron of a $7 \mathrm{~kb}$ primary transcript C13orf25 in the chromosome 13q31.3 and encodes six highly conserved mature miRNAs [26]. Numerous researches demonstrated that expression of miR-17-92 cluster was markedly up-regulated in multiple solid tumors, showing vital functions in the occurrence and development processes of cancer [27, 28].

Mitofusin (Mfn), encodes by mitofusin genes, is of two kinds of molecules, Mfn1 and Mfn2, exerting influential effects in mitochondrial fusion process [8]. Mitochondrial fusion can protect cells from apoptosis, acting as a repair mechanism of epicyte damages [29]. Mitofusins, as novel cancer-related genes, inhibit cell proliferation and promote cell differentiation [30]. Scholars successfully constructed Mfn2 genes eukaryotic expression vectors $\mathrm{pEGFP}-\mathrm{mfn} 2$ by cloning ORF sequence of Mfn2 into pEGFP-C2. MCF-7 cell proliferation was sharply inhibited after the transfection of pEGFP-mfn 2 vectors and cell arrested in S phase, which might related with the morphology changes of mitochondria [31]. Lowered expression of Mfn2 is an important mechanism in the proliferation of tumors and may be involved in cancer occurrence and development processes. Studies have found that Mfn2 is a potential UBCC tumor suppressor gene, which shows significantly lowered expression in tumor tissues than adjacent non-tumorous tissues and could promote apoptosis and inhibit the proliferation of UBCC cells [11]. In addition, overexpression of Mfn2 inhibited HCC cell proliferation and induced apoptosis by increasing the level of active caspase- 3 and poly (ADP-ribose) polymerase (PARP) cleavage, suggesting that Mfn2 gene may be an important therapeutic target for the treatment of tumors [12]. Unlike accumulated studies on Mfn2, researches on Mfn1 are limited and obscure. 
In the present study, the expression levels of the six members of miR-17-92 cluster in MG-63, Saos-2, U2-OS and hFOB 1.19 cells were detected by fluorescence quantitative RT-PCR, finding that miR-17, miR-19b, miR-20a and miR-19a were remarkably up-regulated in osteosarcoma cells. Differently, previous study found that the expression of the members of miR-17-92 cluster was not changed in human osteosarcoma clinical samples assessed by miRNA microarray [21]. As the lack of researching on miR-19b functions in osteosarcoma cells, miR-19b is selected for the further investigations. Target genes of miR-19b were analyzed by MiRBase, miRanda and TargetScanHuman 6.2, finding that 3' UTR regions on the Mfn1 and Mfn2 genes acted as favorably matched sites, of which Mfn1 was of the highest degree of match. Hence, we evaluated the relationship between miR-19b and Mfn1 and investigate molecular mechanism of both in osteosarcoma cells. Dual-luciferase reporter assay system was used to verify the existence of miR-19b target site on Mfn1 genes. As cells transfected with the reporter vectors served as the control group, luciferase expression level of cells transfected with the vectors cloned with Mfn1 3'UTR sequences decreased by $36 \%$, which was of markedly significant difference. Whereas, luciferase expression changes of cells transfected with the vectors cloned with mutated Mfn1 3'UTR sequences were inapparent. Taken together, miR-19b bound with 3'UTR region of $\mathrm{Mfn} 1$ genes, suggesting that $\mathrm{Mfn} 1$ was one of the target genes of miR-19b. For the examination of interaction between Mfn 1 and miR-19b in osteosarcoma cells, mimics and inhibitors of miR-19b were synthesized and overexpression vectors of Mfn 1 were constructed to transfect into osteosarcoma cells. As shown by western blotting, Mfn 1 expression was down-regulated by miR-19b and increased after inhibition of miR-19b, whereas, up-regulation of Mfn 1 expression was unapparent for simultaneously transfection of miR-19b mimics and Mfn 1 overexpression vectors, further proving the relationship between Mfn 1 genes and miR-19b. Considering that miR-19b targeted 3'UTR region of Mfn1 gene to downregulate the expression of $\mathrm{Mfn} 1$, we examined the influence of such down-regulated effect on osteosarcoma cells. MTS cell proliferation assay showed that cell proliferation was significantly increased with the transfection of miR-19b mimics and was decreased of the group with miR-19b inhibitors, revealing that miR-19b might promote the growth of tumor cells. Furthermore, cell proliferation of the group transfected with both miR-19b mimics and Mfn1 overexpression vectors differed slightly with the control group, while cell growth of the group transfected with Mfn1 vectors alone was inhibited apparently. Herein, we may infer that miR-19b targets Mfn1 to suppress the anti-proliferation effect exerted by Mfn1. For the in-depth exploration of miR-19b and Mfn1 functions, flow cytometry assay was conducted. It was indicated that the DNA content of G1/G0 phase was increased from $47.3 \%$ to $82.0 \%$ with the addition of $\mathrm{miR}-19 \mathrm{~b}$ and to $66.6 \%$ with the transfection of Mfn1 vectors, meanwhile, sub G1 peaks of miR-19b inhibitor group raised from $0.3 \%$ to $15.2 \%$ and sub
G1 peaks of Mfn1 vector group raised from $0.3 \%$ to $11.2 \%$, suggesting a G1/G0 phase cell arrest accompanied with slight cell apoptosis. Transfection of Mfn 1 overexpression vectors and miR-19b inhibitor both significantly triggered early and late apoptosis, and total apoptotic cell death in U2-OS cells. Thereinto, late apoptosis induced by Mfn1 vectors and early apoptosis triggered by miR-19b inhibitor played the leading roles. Moreover, TUNEL-DAPI co-staining assay showed DNA fragmentation and morphological changes of nuclei in the miR-19b inhibitor group and Mfn 1 vector group, implying that the reduction of miR-19b and increase of Mfn 1 promoted the apoptosis of tumor cells. Overall, up-regulation of Mfn1 induced by inhibition of miR-19b facilitates the apoptosis of osteosarcoma cells. We further investigated miR-19b and Mfn1 functions on tumor cell invasion and metastasis. Transwell assay was used to determine the effect of Mfn1 vector and miR-19b inhibitor on MG-63 cell invasion. The results showed that cell invasion was dramatically inhibited both in the Mfn1 vector group and miR-19b inhibitor group, indicating that the Mfn1 played important roles in the inhibition of MG-63 cell invasion.

In conclusion, Mfn1 inhibits proliferation, invasion and metastasis of osteosarcoma cells and promotes apoptosis and cell cycle arrest. While miR-19b targets the 3'UTR sequences of Mfn1 genes and inhibits the expression of Mfn1, thus promotes cancer cell growth. Since miR-19b was significantly up-regulated in osteosarcoma cells, miR-19b inhibitor or Mfn1 expression promoter may serve as new ways for the discovery of novel anti-cancer therapiesand the results from the present study maycontribute to the better understanding onthe mechanisms of osteosarcoma occurrence and development.

Supplementary information is available in the online version of the paper.

\section{References}

[1] NISWANDER LM, KIM SY. Stratifying osteosarcoma: minimizing and maximizing therapy. Curr Oncol Rep 2010; 12: 266-70. http://dx.doi.org/10.1007/s11912-010-0106-3

[2] OTTAVIANI G, JAFFE N. The epidemiology of osteosarcoma. Cancer Treat Res 2009; 152: 3-13.

[3] ZHANG X, YU H, LOU JR, ZHENG J, ZHU H, et al. MicroRNA-19 (miR-19) regulates tissue factor expression in breast cancer cells. J Biol Chem 2011; 286: 1429-35. http://dx.doi. org/10.1074/jbc.M110.146530

[4] HUANG G, NISHIMOTO K, ZHOU Z, HUGHES D, KLEINERMAN ES. miR-20a encoded by the miR-17-92 cluster increases the metastatic potential of osteosarcoma cells by regulating Fas expression. Cancer Res 2012; 72: 908-16. http://dx.doi.org/10.1158/0008-5472.CAN-11-1460

[5] BAUMHOER D, ZILLMER S, UNGER K, ROSEMANN $\mathrm{M}$, ATKINSON MJ, et al. MicroRNA profiling with correlation to gene expression revealed the oncogenic miR-17-92 cluster to be up-regulated in osteosarcoma. 
Cancer Genet 2012; 205: 212-9. http://dx.doi.org/10.1016/ j.cancergen.2012.03.001

[6] XU Y, FANG F, ZHANG J, JOSSON S, ST CLAIR WH, et al. $\mathrm{miR}-17^{\star}$ suppresses tumorigenicity of prostate cancer by inhibiting mitochondrial antioxidant enzymes. PLoS One2010; 5: e14356. http://dx.doi.org/10.1371/journal. pone. 0014356

[7] SUGIOKA R, SHIMIZU S, TSUJIMOTO Y. FZO1, a protein involved in mitochondrial fusion, inhibits apoptosis. J Biol Chem 2004; 279: 52726-34. http://dx.doi.org/10.1074/jbc. M408910200

[8] ISHIHARA N, EURA Y, MIHARA K. Mitofusin 1 and 2 play distinct roles in mitochondrial fusion reactions via GTPase activity. J Cell Sci 2004; 117: 6535-46. http://dx.doi. org/10.1242/jcs.01565

[9] SANTEL A, FRANK S, GAUME B, HERRLER M, YOULE RJ, et al. Mitofusin-1 protein is a generally expressed mediator of mitochondrial fusion in mammalian cells. J Cell Sci 2003; 116: 2763-74. http://dx.doi.org/10.1242/jcs.00479

[10] RYU SW, CHOI K, PARK JH, PARK YM, KIM S, et al. Mitofusin 1 inhibits an apoptosis-associated amino-terminal conformational change in Bax, but not its mitochondrial translocation, in a GTPase-dependent manner. Cancer Lett 2012; 323: 62-8. http://dx.doi.org/10.1016/j.canlet.2012.03.038

[11] JIN B, FU G, PAN H, CHENG X, ZHOU L, et al. Anti-tumour efficacy of mitofusin-2 in urinary bladder carcinoma. Med Oncol 2011; 28 Suppl 1: S373-80. http://dx.doi.org/10.1007/ s12032-010-9662-5

[12] WANG W, ZHOU D, WEI J, WU Z, CHENG X, et al. Hepatitis $B$ virus $X$ protein inhibits p53-mediated upregulation of mitofusin-2 in hepatocellular carcinoma cells. Biochem Biophys Res Commun 2012; 421: 355-60. http://dx.doi. org/10.1016/j.bbrc.2012.04.015

[13] YU J, OHUCHIDA K, MIZUMOTO K, FUJTA H, NAKATA $\mathrm{K}$, et al. MicroRNA miR-17-5p is overexpressed in pancreatic cancer, associated with a poor prognosis, and involved in cancer cell proliferation and invasion. Cancer Biol Ther 2010; 10: 748-57. http://dx.doi.org/10.4161/cbt.10.8.13083

[14] XU XM, WANG XB, CHEN MM, LIU T, LI YX, et al. MicroRNA-19a and -19b regulate cervical carcinoma cell proliferation and invasion by targeting CUL5. Cancer Lett 2012; 322: 148-58. http://dx.doi.org/10.1016/j.canlet.2012.02.038

[15] YANG J, ZHANG W. New molecular insights into osteosarcoma targeted therapy. Curr Opin Oncol 2013; 25: 398-406. http://dx.doi.org/10.1097/CCO.0b013e3283622c1b

[16] GOUGELET A, PISSALOUX D, BESSE A, PEREZ J, DUC A, et al. Micro-RNA profiles in osteosarcoma as a predictive tool for ifosfamide response. Int J Cancer 2011; 129: 680-90. http://dx.doi.org/10.1002/ijc.25715

[17] ESSA S, DENZER N, MAHLKNECHT U, KLEIN R, COLLNOT EM, et al. Challenge and promise: the role of miRNA for pathogenesis and progression of malignant melanoma. Clin Epigenetics 2010; 1: 7-11. http://dx.doi.org/10.1007/s13148010-0001-7

[18] HE J, ZHANG JF, YI C, LV Q, XIE WD, et al. miRNA-mediated functional changes through co-regulating function related genes. PLoS One 2010; 5: e13558. http://dx.doi.org/10.1371/ journal.pone.0013558

[19] JETHWA K, WEI J, MCENERY K, HEIMBERGER AB. miRNA-mediated immune regulation and immunotherapeutic potential in glioblastoma. Clin Investig (Lond) 2011; 1: 1637-1650. http://dx.doi.org/10.4155/cli.11.159

[20] JONES KB, SALAH Z, DEL MARE S, GALASSO M, GAUDIO E, et al. miRNA signatures associate with pathogenesis and progression of osteosarcoma. Cancer Res 2012; 72: 1865-77. http://dx.doi.org/10.1158/0008-5472.CAN-11-2663

[21] MAIRE G, MARTIN JW, YOSHIMOTO M, CHILTONMACNEILL S, ZIELENSKA M, et al. Analysis of miRNA-gene expression-genomic profiles reveals complex mechanisms of microRNA deregulation in osteosarcoma. Cancer Genet 2011; 204: 138-46. http://dx.doi.org/10.1016/ j.cancergen.2010.12.012

[22] IORIO MV, VISONE R, DI LEVA G, DONATI V, PETROCCA $\mathrm{F}$, et al. MicroRNA signatures in human ovarian cancer. Cancer Res 2011; 67: 8699-707. http://dx.doi.org/10.1158/0008-5472. CAN-07-1936

[23] GAROFALO M, QUINTAVALLE C, DI LEVA G, ZANCA C, ROMANO G, et al. MicroRNA signatures of TRAIL resistance in human non-small cell lung cancer. Oncogene 2008; 27: 3845-55. http://dx.doi.org/10.1038/onc.2008.6

[24] HARTING MT, BLAKELY ML. Management of osteosarcoma pulmonary metastases. Semin Pediatr Surg 2006; 15: 25-9. http://dx.doi.org/10.1053/j.sempedsurg.2005.11.005

[25] CALDAS C, BRENTON JD. Sizing up miRNA as cancer genes. Nat Med 2005; 11 (7): 712-714. http://dx.doi.org/10.1038/ $\underline{\mathrm{nm} 0705-712}$

[26] RANJI N, SADEGHIZADEH M, SHOKRGOZAR MA, BAKHSHANDEH B, KARIMIPOUR M, et al. miR-17-92 cluster: an apoptosis inducer or proliferation enhancer. Mol Cell Biochem 2013; 380: 229-38. http://dx.doi.org/10.1007/ s11010-013-1678-7

[27] MATSUBARA H, TAKEUCHI T, NISHIKAWA E, YANAGISAWA K, HAYASHITA Y, et al. Apoptosis induction by antisense oligonucleotides against miR-17-5p and miR-20a in lung cancers overexpressing miR-17-92. Oncogene 2007; 26: 6099-105. http://dx.doi.org/10.1038/sj.onc.1210425

[28] YAN HL, XUE G, MEI Q, WANG YZ, DING FX, et al. Repression of the miR-17-92 cluster by 53 has an important function in hypoxia-induced apoptosis. EMBO J 2009; 28: 2719-32. http://dx.doi.org/10.1038/emboj.2009.214

[29] PERFETTINI JL, ROUMIER T, KROEMER G. Mitochondrial fusion and fission in the control of apoptosis. Trends Cell Biol 2005; 15: 179-83. http://dx.doi.org/10.1016/ j.tcb.2005.02.005

[30] XIA Y, WU Y, HE X, GONG J, QIU F. Effects of mitofusin-2 gene on cell proliferation and chemotherapy sensitivity of MCF-7. J Huazhong Univ Sci Technolog Med Sci 2008; 28: 185-9. http://dx.doi.org/10.1007/s11596-008-0218-2

[31] MA L, LIU Y, GENG C, QI X, JIANG J. Estrogen receptor beta inhibits estradiol-induced proliferation and migration of MCF-7 cells through regulation of mitofusin 2. Int J Oncol 2013; 42: 1993-2000. 